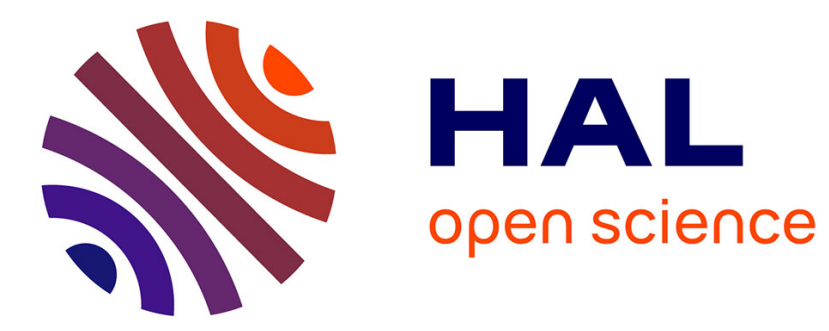

\title{
Militer pour survivre
}

François Godicheau

\section{To cite this version:}

François Godicheau. Militer pour survivre: Lettres d'anarchistes français emprisonnes à Barcelone (1937-1938). Sociétés \& Représentations, 2002, Histoire et archives de soi, 1 (13), pp.137-150. 10.3917/sr.013.0137 . halshs-01552761

\section{HAL Id: halshs-01552761 https://shs.hal.science/halshs-01552761}

Submitted on 11 Oct 2017

HAL is a multi-disciplinary open access archive for the deposit and dissemination of scientific research documents, whether they are published or not. The documents may come from teaching and research institutions in France or abroad, or from public or private research centers.
L'archive ouverte pluridisciplinaire HAL, est destinée au dépôt et à la diffusion de documents scientifiques de niveau recherche, publiés ou non, émanant des établissements d'enseignement et de recherche français ou étrangers, des laboratoires publics ou privés. 


\section{MILITER POUR SURVIVRE \\ Lettres d'anarchistes français emprisonnés à Barcelone (1937-1938) \\ François Godicheau}

\section{Publications de la Sorbonne | Sociétés \& Représentations}

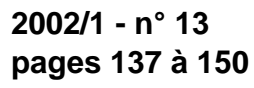

ISSN 1262-2966

Article disponible en ligne à l'adresse:

http://www.cairn.info/revue-societes-et-representations-2002-1-page-137.htm

Pour citer cet article :

Godicheau François, « Militer pour survivre » Lettres d'anarchistes français emprisonnés à Barcelone (1937-1938), Sociétés \& Représentations, 2002/1 n 13, p. 137-150. DOI : 10.3917/sr.013.0137

Distribution électronique Cairn.info pour Publications de la Sorbonne.

(c) Publications de la Sorbonne. Tous droits réservés pour tous pays.

La reproduction ou représentation de cet article, notamment par photocopie, n'est autorisée que dans les limites des conditions générales d'utilisation du site ou, le cas échéant, des conditions générales de la licence souscrite par votre établissement. Toute autre reproduction ou représentation, en tout ou partie, sous quelque forme et de quelque manière que ce soit, est interdite sauf accord préalable et écrit de l'éditeur, en dehors des cas prévus par la législation en vigueur en France. II est précisé que son stockage dans une base de données est également interdit. 


\section{MILITER POUR SURVIVRE}

LETTRES D’ANARCHISTES FRANCAAS
EMPRISONNÉS Ã BARCELONE (1937-1938)

François Godicheau

"Mon vieux copain Fortin... ", écrit Félix Danon depuis la prison de Barcelone, entre juin 1937 et juin 1938. Secrétaire adjoint de la section française de la Confederación Nacional del Trabajo (CNT), il est, avec de nombreux autres anarchistes, en prison dans l'Espagne républicaine, cette Espagne qui lutte contre Franco. Il ne comprend pas tout. Dans les lettres qu'il rédige à l'adresse du secrétaire de la section française, Fernand Fortin, il commente la vie politique de la prison et celle du dehors, il maintient active sa pensée, il reste un militant, comme s'il était dehors, comme si cet emprisonnement n'était qu'une mauvaise plaisanterie, une erreur.

Félix Danon est un idéaliste, "un bohème illuminé " comme il le confesse dans une lettre à son avocat; ses lettres nous introduisent directement, au-delà de la prison ellemême, dans l'intimité d'un engagement militant fort, fidèle à lui-même, investi quotidiennement dans une pratique politique qu'il tente de maintenir derrière les barreaux. Pourtant, ce n'est ni un penseur, ni un dirigeant, c'est un militant certes instruit et pourvu de quelque expérience, mais un militant de base.

Samuel Kaplan, autre anarchiste français emprisonné en Espagne, autre militant de base également, écrit lui aussi à Fernand Fortin. Il est le personnage malheureux d'une tragique odyssée qui s’achève par la mort. Il est moins loquace, moins porté sur l'autodérision et l'humour noir, mais sa détresse et sa rage nous font toucher du doigt, peutêtre plus directement encore, le cœur d'un engagement.

Ce qui caractérise ces correspondances, c'est qu'il y est question d'un idéal trahi: deux hommes partis combattre pour une cause se retrouvent en prison aux côtés de leurs ennemis, les " fascistes ", enfermés par cette Espagne de la révolution à laquelle ils ont cru. L'amertume de cette trahison s'exprime différemment dans l'un et l'autre cas. Chez Danon, elle s'exprime par le maintien de l'activité politique, chez Kaplan par le cri de détresse. Dans les deux cas, on peut la lire dans les mots de l'époque, écrits sur le moment, dans les conditions de l'action elle-même. Il ne s'agit pas de souvenirs écrits quelques années après ou provoqués bien plus tard par un enquêteur. Leur immédiateté 
fait leur valeur: ces correspondances permettent à la fois de renseigner un épisode essentiel de la guerre civile - la répression intérieure contre les anarchistes - et de restituer à l'étude d'un conflit dramatique toute sa charge humaine, ces mots irremplaçables de ceux qui souffrent ${ }^{1}$.

\section{Un conflit interne à la guerre civile}

Le soulèvement militaire et antirépublicain commencé les 17,18 et 19 juillet 1936 dans toutes les garnisons d'Espagne avait échoué dans les principales villes, dont Barcelone, et provoqué en retour une révolution, celle-là même que les insurgés prétendaient éviter. La guerre civile contre ceux qui allaient bientôt s'appeler les franquistes se fit, dans les premiers temps, avec des milices ouvrières organisées par les partis du Front Populaire et les syndicats, pendant qu'à l'arrière, terres et usines étaient collectivisées et réquisitionnées. Le pouvoir de l'État fut presque entièrement éliminé, en particulier en Catalogne, région où les anarchistes étaient majoritaires dans le mouvement ouvrier. L'absence de centralisation des multiples comités révolutionnaires et l'engagement des partis du Front Populaire pour reconstruire le pouvoir d'État, finit par créer une situation où l'un des deux pouvoirs devait disparaittre2.

Pendant l'automne 1936 et le printemps 1937, la tension monte à Barcelone entre les partisans de "la guerre et la révolution ", anarchistes de la Confédération Nationale du Travail (CNT) et de la Fédération Anarchiste Ibérique (FAI), communistes oppositionnels du Parti Ouvrier d'Unification Marxiste (POUM), et leurs adversaires, pour lesquels la seule priorité était de gagner la guerre, le Parti Socialiste Unifié de Catalogne (PSUC), adhérent à la III internationale, et la Gauche Républicaine Catalane (ERC), parti nationaliste majoritaire dans la région depuis l'instauration de la République. En mai 1937, une véritable guerre civile à l'intérieur de la première éclate à Barcelone, entre les partisans de la Révolution et leurs adversaires. Les combats font plusieurs centaines

1. Félix Danon est arrêté le 13 juin 1937 pour avoir, durant les événements de mai, occupé le « Casal del Metge ", siège de l'association professionnelle des médecins, sur l'avenue Durruti, et de là, participé à la défense du local du Comité Régional de la CNT-FAI, assiégé par la police. Son procès a lieu le 9 juin 1938. Il est déclaré non coupable et relâché. Il reste pendant quelques mois à Barcelone pour aider les prisonniers restés dans la prison Modelo. Samuel Kaplan, arrêté en février 1938, sans motif officiel, par le Servicio de Información Militar (SIM), contre-espionnage et police militaire, est encore en prison en janvier 1939, quinze jours avant la chute de Barcelone. Sa situation est vite illégale puisque la détention administrative ne peut excéder 30 jours.

2. Parmi la très importante bibliographie sur ce conflit, on peut consulter, en français, Pierre Broué et Édouard Témine, La Révolution et la guerre d'Espagne, Paris, Minuit, 1961, et Henri Thomas, La Guerre d'Espagne, Paris, Robert Laffont, 1985. En espagnol et en catalan, on peut consulter B. Bolloten Revolución y contrarrevolución en España (1936-1939), Madrid, Alianza, 1995, et P. Pages, I. Blanch, La Guerra civil espanyola a Catalunya (1936-1939), Barcelona, Libres de la Frontera, 1997.

François Godicheau, "Militer pour survivre... », S. \& R., n 13, Avril 2002, pp. 137-150. 
de morts et sonnent le glas de l'influence anarchiste. Les corps de police où ils sont majoritaires sont dissous, toutes les armes sont réquisitionnées, la légalité républicaine triomphe et les avancées de la révolution sont remises en cause dans tous les domaines 3 .

Ce changement de conjoncture s'accompagne d'un mouvement répressif important contre une partie des effectifs militants de la CNT et de la FAI. La persécution soufferte par le POUM est relativement connue (le parti est dissout, ses locaux fermés, ses journaux interdits, plusieurs centaines de militants emprisonnés, des dirigeants inculpés de haute trahison), le martyr de son dirigeant Andrés Nin, mort aux mains du NKVD, la police politique soviétique ${ }^{4}$, en est le symbole. Le vaste mouvement d'arrestations qui jette en prison des milliers de libertaires entre mai 1937 et août 1938 est en revanche fréquemment oublié; il touche le plus souvent de simples militants, des activistes des campagnes, des quartiers de Barcelone ou des miliciens de retour du front ${ }^{5}$. Parmi eux, quelques centaines de militants étrangers venus s'engager dans les colonnes anarchistes ou dans la colonne du POUM, anarchistes français, italiens, allemands, communistes oppositionnels de tous les pays6.

Les lettres de Danon et de Kaplan, retrouvées à l'Institut International d'Histoire Sociale (IISH) d'Amsterdam7', font partie d'un ensemble plus vaste de correspondance de ces "prisonniers antifascistes ", ainsi qu'ils s'appelaient eux-mêmes, lettres collectives et individuelles, écrites depuis diverses prisons catalanes et aragonaises, et qui constituent une documentation précieuse à plus d'un titre. Elles sont une voix originale dans le concert politique de l'arrière républicain; elles contribuent toutes à l'expression d'un courant politique qui ne prend quasiment jamais la parole de façon directe. Contre le cours des événements politiques, contre la remise en cause de la révolution de juillet 1936, elles parlent aussi contre les comités de la CNT, et contre la Commission juridique chargée par celle-ci de les défendre. Elles sont une voix qui n’est pas passée au tamis de l'organisation et de la discipline politique.

3. M. Cruells, Els fets de maig. Barcelona 1937, Barcelona, Editorial Joventut, 1970.

4. Sur cette persécution fameuse mais encore mal connue, voir B. Bolloten, Revolución y contrarrevolución en España, op. cit, pp. 761-794, et A. Suarez, El proceso contra el POUM. Un episodio de la revolución española, Paris, Ruedo Ibérico, 1974, J. Gorkin, El proceso de Moscou en Barcelona. El sacrificio de Andrés Nin, Paris, Aymà, 1974, et V. Alba (dir.), El proceso del POUM, [actes du procès], Lerna, Barcelona, 1989. 5. Je renvoie sur ce point à ma thèse Répression et ordre public en Catalogne pendant la guerre civile, thèse d'histoire, EHESS, 2001.

6. Sur les volontaires étrangers en Espagne, Rémi Skoutelsky, L'Espoir guidait leurs pas. Les volontaires français dans les Brigades internationales (1936-1939), préface d'Antoine Prost, Paris, Grasset, 1998, et Castells Andreu, Las Brigadas Internacionales en la Guerra de España, Barcelona, Ariel, 1974. Sur les anarchistes français et la guerre civile espagnole : D. Berry, "French anarchists in Spain, 1936-1939 ", French History, Oxford University Press, vol. 3, n4, déc. 1989, pp. 442-465.

7. International Institute of Social History, fonds CNT-FAI, série FAI-PE, cartons 18 et 57. On compte une trentaine de pièces pour Félix Danon et une dizaine pour Samuel Kaplan.

François Godicheau, «Militer pour survivre... », S. \& R., n 13, Avril 2002, pp. 137-150. 
Les deux correspondances ici présentées sont complémentaires. Celle de Félix Danon est riche et diverse: lettres, comptes rendus de réunions, affichettes, dessins, reçus de colis, proclamations diverses. Elle permet de reconstituer le quotidien de la vie des « prisonniers antifascistes " dans les deux premières galeries de la Modelo, qui en comptait six. Elle permet de suivre le processus d'auto-identification et de reconnaissance d'un groupe politique à l'intérieur de la prison. La politisation du quotidien carcéral dont elle offre l'illustration découvre ce qui sous-tend toute l'activité politique de l'individu Danon: la force que lui donne l'exercice d'une responsabilité politique. Ces lettres, à l'instar des Mémoires de nombreux prisonniers politiques fameux, illustrent le fait que leur survie comme individus peut être liée au maintien d'une activité collective et critique, militante. L'histoire de Samuel Kaplan résume ce drame et exprime ce que le sang-froid de Danon parvient à cacher le plus souvent. À l'optimisme de l'un répondent l'amertume et le désespoir de l'autre, flétri dans son idéal, perdant jusqu'au bout.

\section{Une fenêtre sur la vie politique des "prisonniers antifascistes"}

À l'intérieur de la Modelo de Barcelone8, les prisonniers de la CNT, de la FAI, des Jeunesses Libertaires (JJLL) et du POUM avaient reconstitué une communauté politique qu'ils appelaient les "prisonniers antifascistes ", par opposition aux prisonniers franquistes et aux droits communs qui occupaient les deux autres tiers de la prison. Cette expression, à laquelle faisait parfois concurrence celle de "prisonniers révolutionnaires ", servait à dénoncer l'existence, en pleine "Espagne antifasciste ", de prisonniers politiques. Elle était à la fois légitimante pour le groupe et déligitimante pour les organisations du Front populaire antifasciste. De fait, l'unité réelle de toutes les organisations (moins le POUM, dissout), ne put se réaliser qu'au printemps 1938, avec la libération de la plupart des antifascistes.

L'activité politique menée dans cette prison était donc un élément très important de la vie de l'arrière en Catalogne, même si elle était presque ignorée de la rue. La correspondance des prisonniers est le seul moyen de la reconstituer ${ }^{9}$. On suit, à travers la documentation de Danon, la constitution en juin 1937 du Comité intérieur des prisonniers de la première galerie, représentant les détenus anarchistes. Un autre comité, du POUM, fonctionnait dans la deuxième galerie.

Un procès verbal manuscrit de treize pages, joint à la correspondance, permet de

8. Ce nom de Prison modèle fut donné à toutes les prisons modernes, pourvues de cellules individuelles, qui furent construites entre 1860 et 1905.

9. Un aperçu cependant dans P. Pages, I. Blanch, La Presò model de Barcelona. Historia d’un centre penitenciari en temps de guerra, Barcelona, Abadia de Montserrat, 1996.

François Godicheau, «Militer pour survivre... », S. \& R., n 13, Avril 2002, pp. 137-150. 
suivre les débats de l'une des assemblées générales réunissant tous les prisonniers antifascistes, et où Danon officiait comme secrétaire. Le 4 juillet 1937: après une courte présentation d'un responsable du Comité, une présidence de séance est élue, comme dans toute réunion syndicale. Le premier sujet abordé est l'état des comptes, qui fait l'objet d'un rapport de la part du trésorier du comité. On passe ensuite à l'élection d'un nouveau Comité, et à la discussion sur les initiatives politiques prises par la précédente assemblée. Lecture est donnée d'une lettre collective adressée le 17 juin à la direction de la CNT, protestant contre la répression, et menaçant d'entamer une grève de la faim si les prisonniers antifascistes n'étaient pas libérés sans délai. Le document est reproduit intégralement dans le procès verbal, ainsi que la réponse du Comité Régional de la CNT et une autre lettre, adressée au directeur de la prison. Après avoir approuvé la gestion politique du Comité, l'assemblée passe à la discussion d'autres sujets.

Cette semi-liberté des " prisonniers antifascistes » peut surprendre. Détenus spéciaux, ils avaient droit à un traitement spécial: durant le jour, les cellules étaient ouvertes et les prisonniers pouvaient se réunir dans les couloir et les cours. Le contrôle de cette partie de la prison était presque entièrement assuré par ce Comité, qui filtrait les entrées et répartissait les prisonniers en fonction de leur appartenance politique. L'ouverture de la prison était telle que pendant l'hiver, nous apprend Danon, les prisonniers brûlaient les portes des cellules pour se chauffer et cuisiner les aliments reçus de l'extérieur, complément indispensable du « rata » de misère distribué par la prison. Au fil des mois, on voit cependant cette liberté se restreindre, et le Comité être de plus en plus discret et plus soumis à l'autorité carcérale. Le 28 janvier, Danon note: « Ici ce n'est plus comme avant, on nous serre la vis chaque jour un peu davantage. Nous avons même une petite garnison de gardes d'assaut ici... $» 10$.

Cette prise de parole des prisonniers, à travers des assemblées générales et à travers des Comités, apparaît dans les lettres de Danon comme l'instrument d'un bras de fer avec l'administration judiciaire et pénitentiaire (comme c'est le cas dans toute revendication politique collective en prison), mais aussi avec la direction de la CNT.

Le premier aspect est le plus évident: on le trouve dans la lettre collective adressée au directeur de la prison et reproduite dans le procès verbal du 4 juillet. Il y était simplement prévenu de la future grève de la faim. Plus loin, dans la réunion, les prisonniers abordent la question des communications avec l'extérieur et discutent des propositions précises à faire au directeur. Ils comparent leur situation avec celle des détenus " fascistes " et avec leurs expériences passées de prisonniers politiques. En fonction de cela, ils décident d'adresser un certain nombre d'exigences précises au directeur, accompagnées d'un ultimatum: « Disons au directeur que si après quelques heures, nous n'avons pas ce

10. IISH, FAI-PE, 18. Les Gardes d'assaut étaient l'un des deux principaux corps des forces de sécurité reconstitués par la République (le deuxième étant le corps des Carabiniers). Il s'agissait d'un corps militarisé, à l'image de l'ancienne Garde Civile, dissoute en juillet 1936.

François Godicheau, "Militer pour survivre... », S. \& R., n 13, Avril 2002, pp. 137-150. 
que nous demandons, ce que les fascistes, eux, ont, nous nous refuserons à toute communication. Et en avant pour la protestation! »11.

Le deuxième bras de fer oppose les prisonniers à la direction de la CNT. De nombreuses allusions de Danon aux discussions qui ont cours dans la prison le montrent, comme ses conversations avec Jaime Balius en particulier, le leader du groupe anarchiste radical des "Amis de Durruti » (dont la direction de la CNT avait demandé l'exclusion, rarement appliquée à la base par les syndicats). On apprend que Balius, honni par la direction de la CNT-FAI, est très apprécié parmi les prisonniers anarchistes, ce qui confirme l'idée que son radicalisme est très bien reçu parmi la base militante à l'extérieur de la prison ${ }^{12}$. On trouve dans les lettres des aveux très intéressants comme cette confidence faite à Fortin, " tout le coup de l'Olympia a été monté par nos Comités, ici, en prison ${ }^{13}$. Quelques jours auparavant, les dirigeants anarchistes, dans un meeting organisé par la CNT au théâtre de l'Olympia, avaient été hués et sifflés, et la salle avait crié son exigence de la libération des prisonniers.

Le conflit avec la direction de la CNT repose sur la défense des prisonniers, qu'elle veut limiter aux aspects techniques et juridiques. Eux veulent une défense politique, et c'est aussi pour cela qu'ils se constituent en collectif, qu'ils s'imposent une discipline d'organisation autour du Comité intérieur, ce qui permet à celui-ci, d'après Danon, de tenir la dragée haute à la Commission juridique de la CNT, organe technique créé pour servir de fusible entre les prisonniers et la direction. Au-delà, le conflit porte sur l'évolution politique de la CNT, les prisonniers n'acceptant pas que celle-ci transige avec les principes de l'apolitisme et de la défense de la révolution.

\section{La politisation du quotidien: rester des militants}

Un rapport écrit après sa sortie de prison par Danon résume toute l'activité menée, pendant les douze mois qu'il a passés derrière les barreaux, par le groupe des anarchistes français en prison ${ }^{14}$. Tout y est considéré sous l'angle politique: non seulement les révoltes pour exiger la liberté et les tentatives d'évasion collective des prisonniers antifascistes, mais aussi les mutineries pour le pain, les obsèques d'un prisonnier tué par un garde, les opérations de résistance passive (grève des parloirs, etc.).

11. Doc. cit. IISH, FAI-PE, 57 A.

12. Les Amis de Durruti était un regroupement anarchiste animé, entre autres, par Jaime Balius, et contestant la politique de conciliation avec ses adversaires politiques menée par la direction de la CNT. Ils préconisaient, contre les communistes, les socialistes et les républicains, la prise du pouvoir par les organisations révolutionnaires. Leur journal clandestin, El Amigo del Pueblo, était lu attentivement par la base militante de la CNT, qui se refusait en général à appliquer l'ordre d'exclusion prononcé contre les Amis de Durruti par la direction.

13. Lettre du 23 juillet.

14. Rapport sur nos activités à la Carcel Modelo, 11 p., c.a. juillet 1938. IISH, FAI-PE, 57 A.

François Godicheau, «Militer pour survivre... », S. \& R., n 13, Avril 2002, pp. 137-150. 
En vérité, dans la correspondance au quotidien, on observe une préoccupation constante pour le maintien d'un sens politique. Cette préoccupation prend deux directions: la sélection des « bons antifascistes » et la gestion matérielle de la solidarité venant de l'extérieur, thèmes d'ailleurs très liés.

Le tri des prisonniers, entre ceux qui méritaient le distinctif "d'antifascistes » et les autres, était fait par les comités intérieurs des deux galeries, puis par le comité unifié CNT-POUM. L'affaire était importante: il s'agissait d'empêcher que des criminels de droit commun, des " voyous et trafiquants » aux motivations privées puissent bénéficier de la protection que constituait l'appartenance aux collectifs des " antifascistes » et, audelà, de la défense juridique organisée par la CNT, sans parler de la solidarité matérielle des syndicats (les syndicats qui reconnaissaient des prisonniers dans la Modelo devaient leur verser le salaire qu'ils auraient perçu au dehors, pour qu'ils puissent " cantiner » et leur famille survivre).

Cette tâche était difficile: comment distinguer un assassin dont les motivations étaient privées et un " camarade " accusé d'avoir assassiné des personnes de droite en août 1936 pour le compte du Comité révolutionnaire du village? Comment distinguer le voleur de celui qui appliquait des réquisitions? L'appartenance au syndicat depuis une date antérieure au 19 juillet 1936 était un critère, la connaissance personnelle des uns et des autres en était un autre. En fait, les prisonniers revendiquaient l'exclusivité du droit de décider, en dernier ressort, qui appartenait à leur collectif, y compris contre la Commission juridique de la CNT. Celle-ci voulait être beaucoup plus exigeante sur les personnes à défendre, pour parer aux accusations émanant des adversaires des anarchistes, de défendre tous les malfaiteurs et les embusqués porteurs d'une carte syndicale.

Ce travail quotidien et ces difficultés sont parfaitement illustrés dans la correspondance de Félix Danon. Lié au Comité intérieur des deux galeries, il était particulièrement chargé de l'examen des nouveaux arrivants étrangers, français en particulier. Cette autonomie correspondait aussi à une différence de traitement entre Espagnols et étrangers: ceux-ci étaient relativement mal défendus par la CNT, même quand ils étaient anarchistes. L'organisation était méfiante et ne voulait être piégée par personne, surtout face à un adversaire communiste toujours prêt à l'accuser de couvrir des espions. Ceux qui en pâtissaient le plus étaient les anarchistes allemands et italiens. Ceux-ci, nous apprend Danon, durent faire appel à la Ligue des Droits de l'Homme et à des organisations socialdémocrates en exil, devant le peu d'empressement de l'organisation libertaire espagnole à les défendre.

Les Français, bien que n'étant pas apatrides (comme c'était le cas de tous les " antifascistes " ressortissants de pays totalitaires, qui n'avaient nulle part où se réfugier), devaient tout aux efforts de Fortin, sur le plan matériel comme sur le plan juridique. On trouve à plusieurs reprises des allusions directes de Danon ou des paroles rapportées d'autres détenus, sur ce traitement «à part » des étrangers.

Danon commença par transmettre des listes de militants français prisonniers dans les deux premières galeries, avec le peu de renseignements disponibles sur leur situation au 
regard de la justice. La plupart ne dépendaient que de la police et n’étaient pas inculpés de quoi que ce soit. En dépit de ce fait, ils pouvaient rester plusieurs mois en détention administrative, avant d'être expulsés et reconduits à la frontière (Danon signale à deux reprises le départ d'une caravane d'étrangers). Mais bien vite, dans le quotidien des lettres, des querelles de la prison et des arrivées de "nouveaux ", les considérations politiques sur les " camarades" se mêlèrent à d'autres, d'ordre plus personnel.

Le Comité des Internationaux Antifascistes repose dans les faits sur Félix Danon et deux ou trois autres militants qui, en fonction des discussions et des réactions quotidiennes des uns et des autres, définissent les limites du groupe. La fidélité au sigle CNT est un élément capital pour décider de l'inclusion ou de l'exclusion d'un militant. Certains, au bout de quelques semaines, par opportunisme ou simplement par amertume, paraissent prêts à rejeter l'organisation. Quelquefois, ce péché aux yeux de Danon se double d'un autre, la critique injustifiée ou l'insulte à l'égard de Fortin. C'est le cas de Julien Schwab, un propagandiste français de la CNT, arrêté après une mission à Albacete effectuée pour le compte du Comité Régional de Catalogne. Danon écrit le 14 juillet: "Ici, Schwab mène campagne contre la CNT-FAI et contre toi. Sourdement, certains comme Lacroisille, dit Marseille, se laissent entraîner. J'ai réussi à leur couper les pattes à une réunion d'étrangers (ils ont parlé de "sabotage") »15.

La moralité, le respect de certaines règles de conduite, de dignité, le " puritanisme anarchiste " constituent d'autres critères importants pour décider de l'appartenance au groupe des " antifascistes". La prohibition de la boisson fait partie de ces règles. L'important est de ne pas offrir une image dégradée aux autres prisonniers et à l'administration, ce qui ruinerait le crédit gagné à travers l'organisation politique en Comité. C'est ce que reproche Danon à une trentaine de prisonniers des Brigades Internationales:

Les fameux « 30 " d'Albacete16 trouvent moyen de se saouler sans avoir le... sou. Ils ont déjà vendu foulards et godasses pour pouvoir s'acheter des chopines! Comme on n'a pas voulu de ces loustics dans notre Galerie, ils sont avec des fascistes et des gens de la pègre. Ils la baillent belle à nos adversaires. Ah! oui. Ce que "falangistas » et " requetés " 17 doivent penser des Milices et des miliciens, surtout de la si fameuse «brigade Internationale »! Aujourd'hui, je vais dans leur galerie, non seulement pour les engueuler (ce que j’ai déjà fait hier) mais pour faire comprendre aux fascistes que si ces oiseaux sont là ce n'est ni pour les événements de mai, ni pour leurs opinions trop révolutionnaires. Et qu'on expulse des Milices les ivrognes.

Car les fascistes les regardent avec des yeux farouches et sadiques: ils leur donnent même de l'argent pour s'acheter du vin. Les fascistes clignent de l'œil et leurs bouches grimacent en diaboliques sourires: sans doute pensent-ils qu'on peut acheter pour quelques litres de pinard trente

15. IISH, FAI-PE, $57 \mathrm{~A}$

16. Albacete était le siège de la direction, du camp de formation et de la prison des Brigades.

17. Phalangistes: membres de la Phalange, l'organisation fasciste franquiste. Réquétés: membres des milices carlistes (légitimistes et catholiques intégristes).

François Godicheau, «Militer pour survivre... », S. \& R., n 13, Avril 2002, pp. 137-150. 
"Communistes ", car toute cette racaille aux bras et à la poitrine tatoués se fait passer pour " antifasciste " et " communiste »! - et eux sans doute de se dire que si toute la fameuse "Brigade Internationale » est ainsi: une brigade de soûlards! ! Je ne sais vraiment pas ce qu'on attend pour les renvoyer à leurs bistrots d'au-delà des Pyrénées $($ sic) 18

La défense de l'honneur de la Brigade Internationale et des communistes peut surprendre de la part d'un anarchiste mis en prison à l'occasion d'une lutte entre la CNT et le parti communiste, de la part de quelqu'un qui critique ses propres camarades pour des désaccords qui pourraient sembler minimes en comparaison. En réalité, la logique de Danon n'est pas une logique sectaire qui considère les individus à travers l'étiquette partisane qu'ils portent, communiste, anarchiste, poumiste, trotskiste. Les " prisonniers antifascistes " qui méritent de recevoir l'aide matérielle, morale et juridique de l'organisation sont ceux qui ont lutté et qui se déclarent prêts à continuer à le faire, pour " la guerre et la révolution ». L'engagement dans la guerre civile espagnole a un sens qui ne peut être dilué, encore moins en prison.

La sélection opérée par les Comités (et par Danon), selon divers critères - politiques, personnels, de moralité - a pour but de renforcer la cohésion du groupe et son identité " révolutionnaire " (et même de Juste), hostile à la politique de guerre "réformiste " ou " contre-révolutionnaire » menée par les communistes, les républicains, les policiers et les juges. C'est pour cela que le Comité intérieur de la prison exclut des adhérents de la CNT qui ont commis des forfaits étrangers au mouvement, et accepte de défendre des militants de l'UGT accusés de "crimes révolutionnaires ". C'est ce qui explique aussi que Danon montre une certaine bienveillance à l'égard de plusieurs déserteurs des brigades, quill les aide, même lorsqu'il s'agit de militants du PCF, de la CGT, de la SFIO ou du POUM. Il les interroge pour mesurer leur caractère et leurs motivations.

Ce deuxième cercle de reconnaissance, celui des " prisonniers révolutionnaires ", audelà du premier qui regroupe juste les "copains de la section " (section française de la CNT), permet à ceux qui y sont inclus de bénéficier de l'aide matérielle envoyée par Fortin ou amenée directement à la prison par lui-même ou par ses émissaires: aliments, savon, papier et stylos, cigarettes, journaux.

Laide matérielle n'échappait pas à la politisation. Les journaux étaient lus et commentés en groupe par les prisonniers, qui en réclamaient toujours plus. Leur présence permettait de faire disparaitre dans une certaine mesure les murs de la prison; ainsi les Français de la CNT recevaient et discutaient le bulletin d'information CNT-FAI édité par Fernand Fortin. Ils écrivaient à des journaux anarchistes de France, ils continuaient à participer à la vie militante.

Les aliments aussi faisaient l'objet d'un traitement spécifique, formel et collectif: pour éviter les vols par les gardiens ou d'autres intermédiaires, les paquets envoyés par

18. Lettre du 31 juillet 1937.

François Godicheau, "Militer pour survivre... », S. \& R., n 13, Avril 2002, pp. 137-150. 
Fortin contenaient une liste tapée à la machine et signée19. En retour, les prisonniers devaient envoyer un reçu signé détaillant une nouvelle fois le contenu du colis. Ces reçus étaient numérotés par Danon qui s’inquiétait dans chaque lettre de leur bonne réception.

À l'échelle des Comités de galerie, on remarque des pratiques semblables qui témoignent d'un grand formalisme pour la répartition des biens " collectifs ». Dans le procès verbal de l'assemblée du 4 juillet 1937, Danon reproduit intégralement le rapport financier établi par le trésorier du Comité. Celui-ci comprend un état des comptes le 6 juin, un autre le 24 juin, un dernier le 4 juillet, avec le détail des entrées jour par jour, en liquide ou en nature (le dernier état hebdomadaire comprend des pantalons, des chemises, des caisses de fruits, du café, du sucre, du jambon, des encriers, des stylos, du papier, des timbres), où est précisée l'origine des dons (Commission juridique, tel comité syndical, tel militant). La nature des commandes est discutée en assemblée générale à la suite du vote du quitus pour le trésorier: il s'agit d'encadrer le plus possible la vie matérielle des prisonniers politiques.

De même, les loisirs en prison sont organisés collectivement: des conférences, des débats publics et contradictoires sont annoncés. Le 14 juillet, Danon rapporte: "Laina, du Comité Régional des Jeunesses Libertaires, a fait une belle conférence l'autre jour. Un camarade du POUM lui a fait la contradiction : c'était intéressant. " On trouve aussi dans sa correspondance l'affiche qu'il a dessinée, invitant à un " cycle de conférences » dans le patio de la seconde galerie sur le thème: "Que doit faire la classe travailleuse pour vaincre? "20. Le 26 septembre, un "festival " est même organisé, dont le programme nous est parvenu: on y annonce, après une "présentation par un poète cosmopolite " (Danon lui-même), des " chants régionaux par le plus flamenco de la compagnie ", Rafael Guerra, dit, "Peu de voix ", un intermède comique, d'autres chants, poésies, " contes allemands ", blagues, etc. Le spectacle est signé par deux groupes «Derrière les barreaux de la prison " et "les Microbes ", le tout sous le contrôle du Comité intérieur. Dans une lettre postérieure, Danon parle à Fortin du succès de cette journée et de l'effet sur le «moral des camarades $» 21$.

La vie dans la prison, rythmée par les révoltes et les protestations (en moyenne une par mois), reçoit, dans la plupart de ses aspects, un sens politique. L'identification politique permanente à travers le collectif et l'organisation en Comités permet aux prisonniers de " tenir ", c'est-à-dire, de lutter contre le sentiment de l'absurde, d'être des révolutionnaires en prison un an après le «triomphe de la révolution » en juillet-août 1936. Quand ils écrivent, dans la protestation collective adressée le 17 juin à la direction de la CNT, que « l'arrestation et l'inculpation de ceux qui ont tout donné pour la Révolution, avant et après le 19 juillet [1936], est la plus grande monstruosité que l'on puisse commettre au nom d'un vieux système policier », ils utilisent le mot « monstrueux » dans son

19. Lettre du 28 janvier 1938, IISH, FAI-PE, 18.

20. IISH, FAI-PE, $57 \mathrm{~A}$.

21. Programa del domingo 26 de septiembre, IISH, FAI-PE, 18.

François Godicheau, "Militer pour survivre... », S. \& R., n 13, Avril 2002, pp. 137-150. 
sens premier de " contre nature »: leur situation est le résultat du mariage entre la vieille répression policière bien connue sous les régimes antérieurs et l'Espagne du 19 juillet, celle de la victoire du " peuple » sur le « fascisme ».

\section{L'expression d'une désillusion tragique}

Cette incompréhension, ce sentiment d'avoir été trahi, de l'être en permanence, par tous (non seulement les adversaires politiques, mais aussi l'organisation CNT elle-même, qui refuse de les défendre comme révolutionnaires et s’adapte à la politique de guerre), apparaît rarement dans les lettres de Félix Danon tant la fermeté de sa volonté, la force de son idéalisme sont grandes. Le fait qu'il soit l'organisateur, celui qui a la responsabilité politique de la défense des militants, celui qui pense le collectif avant l'individuel, lui permet sans doute de déployer un optimisme et de maintenir un moral qui apparaissent beaucoup moins solides chez les autres prisonniers. Ses lettres sont la démonstration quotidienne de l'importance vitale de son activité militante en prison: il y engage sa vie et son énergie, il en reçoit un sens qui chaque jour le porte. C'est seulement dans une lettre du 25 mai 1938, adressée à la Commission juridique de la CNT, qu'il laisse affleurer son indignation, alors que beaucoup ont été libérés, et qu'il reste peut-être un peu isolé en prison:

Un camarade qui en mai dernier a préféré défendre les locaux de l'organisation contre les provocateurs, au lieu d'aller tranquillement dormir chez lui, vous le laissez accuser de délits imaginaires et infamants comme "vol à main armée", etc. (...) Je me suis sacrifié pour l'organisation confédérale et anarchiste, je suis en prison depuis près d'un an, payant pour les idioties que d'autres, qui sont en liberté, ont fait. ${ }^{22}$

La correspondance de Samuel Kaplan Milgram, le deuxième correspondant régulier de Fernand Fortin, présente une figure inverse, où domine la détresse et l'indignation. Son histoire est beaucoup plus rocambolesque, exceptionnelle par son tragique.

Français d'origine lituanienne, il affirme être en Espagne depuis 1930 ou 1931, et avoir été naturalisé quatre ans après son arrivée. Cette qualité ne lui est pas reconnue en réalité et le fait d'être apatride, en plus d'anarchiste, lui vaut un surcroît de malheur. Militant de la CNT depuis plusieurs années, il s'est engagé dès juillet 1936 dans les colonnes confédérales parties lutter en Aragon contre les insurgés. Après dix-sept mois sur le front, l'unité d'étrangers de la 26e division, ancienne colonne Durruti, est dissoute et ses éléments congédiés.

Désireux de continuer à se battre, il se présente au bureau de recrutement des Brigades Internationales. Les officiers à qui il a affaire refusent de l'employer au poste

22. Archivo Histórico Nacional-Sección Guerra Civil, Salamanque, Série Politico Social Barcelona, dossier 365 .

François Godicheau, «Militer pour survivre... », S. \& R., n 13, Avril 2002, pp. 137-150. 
qu'il occupait dans son unité: chauffeur de camion. En revanche, ils lui promettent qu'il ira en première ligne avec un fusil, comme fantassin. Là, il prend peur: il est persuadé, comme beaucoup de ses camarades de la CNT-FAI, que dans les unités dirigées par les communistes, les anarchistes servent de chair à canon, et que, quand ils ne sont pas tués par l'ennemi en première ligne, ils sont abattus d'une balle dans le dos. Il refuse alors d'entrer dans les Brigades, quitte le bureau de recrutement avec l'intention de s'engager plutôt dans les unités confédérales que la CNT est en train de mettre sur pied. Mais il est rattrapé par le SIM (Service d'Information Militaire) des Brigades et emprisonné. Nous sommes en février 1938.

Le 12 avril 1938, il écrit d'un wagon-prison stationné sur la voie deux de la Gare de France, à Barcelone, et raconte son histoire. Il est enfermé là avec de nombreux autres CNTistes, mêlés à des prisonniers de guerre franquistes, dans des conditions lamentables. Le 21 avril, il écrit cette fois de la prison de Castelldefels, près de Barcelone. Le 29 avril, il est transféré dans le bateau-prison Uruguay, et de là, il écrit un mot le 21 juin:

Ici dans le bateau, ils ont perdu tout sens humain et si en plus tu protestes, ils te traitent encore pire. C'est mille fois pire qu'en Allemagne dans les camps de concentration. Depuis le 29 avril, je n'ai pas vu le soleil et je manque d'alimentation et de savon pour laver mes vêtements et mon visage. Nous dormons par terre dans la plus grande misère. Je suis désespéré d'être enfermé sans aucune nouvelle de l'organisation ni de ma compagne. ${ }^{23}$

Ce mot, Kaplan le glisse dans une bouteille après avoir écrit dessus: « Je prie les camarades pêcheurs qui trouveront cette lettre d'un anarchiste emprisonné et traité d'une façon pire qu’au temps de la dictature de la porter au Comité national de la CNT, 30 avenue Durruti, section française de Barcelone. Salut et Anarchie ». Miracle des archives, ce message dans la bouteille, trouvé par un marin et amené à la bonne adresse, a été conservé. Il est transmis à Fortin tel quel, accompagné d'un mot de Juan Segarra, responsable de la Commission juridique, expliquant qu'un homme l'ayant trouvé dans une bouteille l'a amené jusqu’à eux.

Dans les mois qui suivent, Kaplan continue son parcours entre les prisons. Dans aucun de ces lieux n'existe un Comité actif de prisonniers, un minimum de vie militante, ni même souvent, un contact avec l'extérieur régulier. En septembre 1938, il est au camp du Pueblo Español, sur la colline de Montjuich, qui domine Barcelone. Le 15, lors d'une tentative d'évasion, il reçoit une balle dans les reins et il est ramené, après un passage à l'hôpital, dans le bateau Uruguay. Le premier octobre, il écrit depuis une autre prison du SIM, connue sous le nom du "séminaire », rue Muntaner: « je suis toujours malade, avec le visage et les jambes enflées à cause du manque d'aliments „24. Le 11 octobre, enfin, il est transféré à la prison d'État de la rue Deu i Mata. Là, il peut commencer à

23. IISH, FAI-PE, 18.

24. 1er oct. 1938, Ibid

François Godicheau, "Militer pour survivre... », S. \& R., n 13, Avril 2002, pp. 137-150. 
recevoir un peu d'aide, quelques aliments, du tabac, du papier. Nous lisons les deux lettres qu'il reçoit de sa compagne, sans ressource à Valence depuis qu'il ne lui envoie plus l'argent de sa solde. Il s'inquiète car elle est enceinte. Son prénom parait incroyable, elle s'appelle Libertad.

En octobre et novembre, il reprend espoir. Il n'a toujours pas vu de juge, on ne lui a toujours pas notifié pourquoi il est enfermé. Son expulsion pose problème car il est apatride. Il se plaint du manque d'efficacité de l'organisation pour la résolution de son cas, mais aussi du froid: en février, le SIM lui a confisqué ses chaussures, et il commence à avoir froid aux pieds à l'heure de la promenade. On voit comment la reprise du lien militant fait revenir le moral: ses paroles sont moins désespérées, il déclare que si, une fois qu'il sera expulsé, l'organisation a besoin de lui, il est prêt à revenir clandestinement. Pourtant, les semaines passent et le seul changement notable est la raréfaction de la nourriture, à un point tel que le 20 décembre, il annonce à Fortin que lui et d'autres prisonniers étrangers, anciens miliciens et interbrigadistes, vont faire une grève de la faim. Tant qu'à mourir de faim, ils veulent convertir leur mort en un dernier geste politique. Cette lettre est l'avant-dernière, de nouveau pleine de détresse. Quand il écrit « même si l'organisation ne montre pas le moindre intérêt pour ma libération, je suis toujours à sa disposition ${ }^{25}$, il s'accroche, malgré le sentiment d'abandon, au lien avec le collectif, qui fait son identité de militant, la dernière chose qu'il puisse perdre. Pourtant, dans cette lettre, il fait part de toute son amertume, de ce sentiment d'avoir été complètement floué que seuls ses mots peuvent dire:

Au cas où je ne recevrais pas de réponse du ministre ni (...) du Directeur Général de la Sécurité, après un délai de 96 heures (...) qui se termine samedi à deux heures, je me vois obligé de déclarer la grève de la faim pour protester contre l'injustice dont je suis victime depuis neuf mois et de la manière qu'a l'Espagne républicaine de payer ses combattants. Je pense la mener jusqu'à la fin, c'est à dire, la liberté ou le cimetière. Parce que je préfere en terminer d'un bon coup plutôt que par une mort lente. À toi Fortin, je te demande que tu me dises si je peux compter sur un quelconque appui de la [Commission] Juridique dans mon cas. Je te demande aussi de m'envoyer 50 ptas parce que je dois 32, 5 ptas pour le papier à lettre, et aussi que tu regardes s'il y a un paquet pour moi car ça fait plus d'un mois que ma sœur m’en a envoyé un.

Maintenant je vais dire quelques mots que ça me fait de la peine de prononcer, oui, c'est triste quand on doit dire ces mots à une organisation à laquelle on a tout donné pendant huit ans. Oui Fortin, l'organisation valait seulement à l'époque, quand ils étaient poursuivis par un Primo de Rivera et un Gil Robles. Mais maintenant qu'ils ont des fauteuils confortables, ça leur fait quelque chose de se bouger par peur que les fauteuils confortables s'enrhument. Ma compagne a demandé au [Comité] National pour moi et la réponse a été que quand je serai inculpé, ils se chargeraient des frais d'avocat et je serai mis en liberté. Ça laisse entendre de quelle manière le [Comité]

25. 20 déc. 1938.

François Godicheau, "Militer pour survivre... », S. \& R., n 13, Avril 2002, pp. 137-150. 
National s'occupe des prisonniers, que moi, après neuf mois enfermé, ils ne savent même pas que je suis juste une pauvre victime d'un secteur politique qui s'appelle fascisme rouge, et je suis en détention administrative et je n'ai aucune inculpation, et je n'aurai pas de jugement (... $)^{26}$

Le 5 janvier 1939, Samuel Kaplan est transféré à la prison militaire du château de Montjuich: le directeur de la prison d'État a décidé de briser le groupe des grévistes de la faim, après quatre morts. Il continue sa grève, il est dans une cellule de condamné à mort. Le 21 janvier, les troupes franquistes entrent à Barcelone. Les prisonniers qui n’ont pu s'échapper sont fusillés. Nulle trace de Kaplan.

Deux anarchistes français combattant contre le franquisme se retrouvent derrière les barreaux républicains. Ils vivent cette contradiction différemment. Le premier a la chance d'être dans une prison où le régime de liberté et la présence de centaines "d'antifascistes " lui permettent de continuer à exercer une activité militante qui l'aide à donner sens à cet épisode de sa vie. Ses lettres sont essentielles pour repérer l'existence et les activités d'un secteur radical de la base anarchiste, qui en prison s'organise et s'exprime de façon autonome, comme il ne peut pas le faire en dehors. Le second, ballotté d'une cellule à l'autre, dans des conditions d'incarcération beaucoup plus difficiles, ne peut presque pas bénéficier de ce soutien. Son parcours de huit mois entre sept prisons, sans jamais voir de juge, révèle l'arbitraire qui règne sur le terrain de l'ordre public.

Au-delà de l'éclairage porté sur les questions de répression et d'ordre public, ces documents valent par l'expression d'un sentiment individuel, qui fait ressortir la vie au cœur des catégories de l'historien, ordre et désordre, partis et organisation. Pour ces deux hommes, la désillusion est forte, mais leur situation de détresse permet d'approcher une conviction et un engagement militant dans un collectif politique (l'organisation CNT) qui les portent jusqu'au bout, l'un vers la liberté, l'autre vers la mort.

26. 20 décembre 1938. Syntaxe et orthographe originales. Ibid. 